DOCTRINA

\title{
El impacto climático de la basura: Análisis normativo de los residuos sólidos, la recuperación de suelos y la minería de rellenos sanitarios
}

\author{
The climatic impact of garbage: Legal analysis of solid waste, \\ recovery and landfill mining
}

Constanza Pelayo Díaz iD

Investigadora independiente, Chile

Izaskun Linazasoro Espinoza

Universidad de Chile y Universidad Católica Silva Henríquez, Chile

RESUMEN Este artículo tiene como objeto analizar la gestión de los residuos sólidos en Chile. Actualmente, es uno de los mayores problemas de la sociedad, tanto en nuestro país como a nivel mundial. Junto con elaborar un diagnóstico y análisis de la normativa aplicable a los residuos y sus problemas asociados, esta investigación —al margen de abordar el concepto de recuperación de suelos y sus diferentes formas de ejecución, con especial énfasis en el denominado landfill mining - busca proponer las bases que permitan su evaluación ambiental junto con determinar su contribución al cambio climático.

PALABRAS CLAVE Residuos sólidos, verted deros, minería de rellenos sanitarios.

ABSTRACT The purpose of this paper is to analyze the solid waste management in Chile. Currently, it is one of the biggest problems of society, in our country and worldwide. In addition to make a diagnosis and analysis of the regulations applicable to waste and its associated problems, this research - beyond from raise the concept of soil recovery and its different forms of execution, with special emphasis on landfill mining - will seek to propose the bases that allow its environmental assessment along with determining its contribution to climate change.

KEYWORDS Solid waste, landfills, landfill mining. 


\section{Introducción}

En 2005, el Protocolo de Kioto reconoció la importancia de los suelos, señalando que las emisiones netas de carbono podrían ser reducidas a través de su acumulación en sumideros terrestres, pues los suelos son «uno de los principales sumideros de carbono de la naturaleza [...] con potencial para incrementar la retención de $\mathrm{CO}_{2}$ » (Ortiz Bernard y otros, 2007: 19). ${ }^{1}$ El problema, es que, si bien el impacto final en la recuperación de suelos resulta positivo para el cambio climático y la disminución de gases de efecto invernadero, el proceso de recuperación tiene consecuencias tanto positivas como negativas que es necesario evaluar a través de un análisis costo-beneficio que permita determinar su eficiencia.

Precisamente, los gases de efecto invernadero, específicamente de metano y el dióxido de carbono proveniente de los vertederos, representan la mayor fuente de impacto en el cambio climático en el sector de residuos (UNEP, 2010). Esta situación cobra especial relevancia a la hora de analizar los datos entregados por la División de Desarrollo Regional de la Subsecretaría de Desarrollo Regional, en un estudio elaborado el año 2018 (Subdere, 2018), en el marco del Programa Nacional de Residuos Sólidos, que identifica 128 sitios operativos para disponer los residuos sólidos domiciliarios en el país, que reciben un total de 7.487.559 de toneladas de desechos al año. Pero aún más preocupante resultan las cifras relativas a los 43 rellenos sanitarios y vertederos que ya cumplieron su vida útil y que, por falta de habilitación de nuevos recintos, siguen recibiendo cerca del $33 \%$ de los residuos sólidos a nivel nacional. La situación es más grave teniendo en cuenta que ninguno de estos estudios considera los vertederos ilegales. De acuerdo con cifras del Ministerio del Medio Ambiente, solo en la región Metropolitana existen cerca de 81 vertederos ilegales y más de 700 microbasurales.

Este diagnóstico permite plantear una tesis inicial: la gestión de residuos en nuestro país es deficiente, razón por la cual se genera un aporte importante de gases de efecto invernadero, así como contaminación de los suelos. Necesitamos alternativas que nos permitan revertir esta situación y que, a su vez, sean atractivas, tanto para los inversores como para los ciudadanos. Considerando este panorama, el landfill mining, o minería de los rellenos sanitarios, aparece como una alternativa de recuperación de áreas en las que existe un vertedero o rellenos sanitarios, y que permite a su vez obtener un producto final derivado de este proceso. En efecto, la minería de rellenos sanitarios puede ser definida como el proceso de extracción de minerales $\mathrm{u}$ otros recursos naturales sólidos, con el fin de que sean reutilizados para generar

1. La retención o secuestro de carbono implica la eliminación del $\mathrm{CO}_{2}$ atmosférico por las plantas y el almacenamiento de carbono fijado como materia orgánica en el suelo. Véase Ortiz Bernard y otros (2007). 
energía o fabricar nuevos productos, a partir de residuos que anteriormente han sido dispuestos enterrándolos en el suelo. Es una técnica originalmente probada en Israel en 1953, pero que ha tenido pocos seguidores.

Las experiencias internacionales son escazas, bastante recientes y aún experimentales (en países como India, Estados Unidos, Alemania y Holanda), y en Chile no se ha implementado, por lo que la práctica internacional merece especial atención. De nacer como una forma de minería metálica y producción de combustible hace más de cincuenta años, su modelo de negocio ha servido incluso para transformar antiguos vertederos en hermosos parques y conjuntos habitacionales para el futuro. Financial Times la ha calificado incluso como «una de las cincuenta ideas que podrían cambiar el mundo».2

Para que pueda ser desarrollada en nuestro país, es necesaria una legislación que haga que esta actividad sea económica, social y ambientalmente sostenible, además de entenderla como complementaria a una política de gestión de residuos que contemple el reciclaje, reutilización y valorización de residuos como primera alternativa, previo a su disposición final. En ese sentido, es necesario analizar si actualmente la legislación chilena permite realizar este tipo de proyectos, y si es considerado dentro del listado de proyectos que deben ingresar al Sistema de Evaluación de Impacto Ambiental de acuerdo con la Ley 19.300.

\section{Diagnóstico: El problema de la basura}

El problema de la basura se ha convertido en una de las mayores preocupaciones del mundo globalizado, principalmente a raíz de la aparición de grandes concentraciones de habitantes en áreas metropolitanas, también de grandes extensiones. Los rellenos sanitarios y vertederos aparecieron en un primer momento como una solución inmediata a un problema sanitario asociado a la vida urbana; sin embargo, con el crecimiento de la ciudad, el uso de estos terrenos ha generado otros tipos de conflictos socioambientales.

No obstante, parece que - al margen de las externalidades negativas inmediatas que conlleva la instalación y funcionamiento de un relleno sanitario- poco se han tomado en cuenta los efectos climáticos que traen consigo: la emisión de gases de efecto invernadero y el aumento de riesgo de contaminación de fuentes de agua subterráneas y superficiales. La preocupación respecto de las consecuencias derivadas de la existencia de este tipo de proyectos aumenta cuando analizamos las cifras del país en torno a la basura.

Pero el problema de la basura no es solamente una cuestión cuantitativa; la ges-

2. Josh Jacobs, «Europe's half a million landfill sites potentially worth a fortune», Financial Times, 8 de enero de 2018, disponible en https://www.ft.com/content/obf645dc-d8f1-11e7-9504-59efdb7oe12f. 
tión y manejo de residuos sólidos se han desarrollado de forma inorgánica a través de nuestra legislación, dependiendo siempre del tipo de residuo. Por ejemplo, respecto de los residuos sólidos domiciliarios, existe poca responsabilidad ciudadana —aunque es creciente- por su disminución y por el fomento al reciclaje. Esto refuerza la necesidad de mayor gestión pública para ayudar al cambio de hábito de la comunidad. Respecto de los residuos sólidos industriales, la responsabilidad ya no es tanto de los organismos públicos ni de los ciudadanos, sino de los privados titulares de proyectos de inversión que los generan o importan.

\section{Diagnóstico cuantitativo del problema de la basura}

La División de Desarrollo Regional de la Subsecretaría de Desarrollo Regional y Administrativo (Subdere) tiene a cargo el Programa Nacional de Residuos Sólidos (PNRS), que consiste en «un programa de inversión pública, cuyo propósito es mejorar las condiciones de salubridad y calidad ambiental de los centros urbanos y rurales del país, a través de la implementación de sistemas integrales y sostenibles para el manejo eficiente de residuos sólidos domiciliarios».3 En el marco de este programa, la autoridad desarrolló, a través de la consultora SGS SIGA, a la cual le encargó este estudio, el informe «Diagnóstico de la situación por comuna y región en materia de RSD [residuos sólidos] y asimilables», de julio de 2018, el que busca ser una herramienta de información actualizada, detallada y de verificación de los sitios de disposición final e instalaciones para la gestión de residuos sólidos.

De acuerdo con este informe, la disposición final es el destino más utilizado en nuestro país para la eliminación de los residuos sólidos domiciliarios y asimilables (RSDyA), y alcanzando el 96,5\%, mientras que el reciclaje solo se realiza respecto del 1,7 \% de los residuos declarados en el Registro de Emisiones y Transferencias de Contaminantes (RETC), y otras alternativas de valorización representan el 1,8\% (Subdere, 2018). Los sitios de disposición final para este tipo de residuos sólidos pueden dividirse en: i) rellenos sanitarios, que son instalaciones de eliminación de residuos sólidos diseñadas, construidas y operadas para minimizar molestias y riesgos para la salud y la seguridad de la población y daños para el medio ambiente, en las cuales los residuos son compactados en capas al mínimo volumen posible y son cubiertas diariamente, cumpliendo con las disposiciones del Decreto Supremo 189/2008; ii) vertederos, que son lugares de disposición final de residuos planificados para ese uso, pero que no cuentan con las medidas sanitarias mínimas establecidas en el decreto mencionado, razón por la cual, en general, son foco de problemas ambientales; y iii) basurales, que son lugares en los que se disponen residuos, ya sea

3. Resolución Exenta 9.187 de 2018 que sustituye la guía operativa del Programa Nacional de Residuos Sólidos, de la Subsecretaría de Desarrollo Regional y Administrativo. Artículo 1, 2. Objetivo general. 
en forma espontánea o programada, sin ningún tipo de control sanitario ni protección ambiental.

A junio de 2018, existen 128 sitios operativos de disposición final de residuos sólidos domiciliarios y asimilables en nuestro país, de los cuales 30 corresponden a rellenos sanitarios, 8 a rellenos sanitarios manuales, ${ }^{4} 52$ a vertederos y 38 a basurales (Subdere, 2018). Si bien existen más vertederos y basurales que rellenos sanitarios, estos reciben el $78,2 \%$ de los residuos sólidos domiciliarios generados a nivel nacional.

De acuerdo a los datos entregados por el Cuarto reporte del estado del medio ambiente en 2018, elaborado por el Ministerio del Medio Ambiente (SINIA, 2018: 69), la generación de residuos a nivel nacional presentó un aumento menor al 1\% entre 2015 y 2016, pasando de 21,1 a 21,2 millones de toneladas. La mayor cantidad fue en residuos no peligrosos, con el $97 \%$ del total, equivalentes a 20,5 millones, que se reparten en un $61,7 \%$ de residuos de origen principalmente industrial y un $36,4 \%$ de origen municipal (o domiciliario). La generación de residuos peligrosos durante el 2016 representó el $3 \%$ a nivel nacional, equivalente en 641,9 mil toneladas.

El mismo año 2016, a nivel nacional la mayor parte de los residuos no peligrosos fue eliminada ( $76,4 \%$, equivalente a 15,7 millones de toneladas), mayoritariamente en rellenos sanitarios, y solo el 23,6\% fue valorizada (SINIA, 2018: 75).

\section{La Ley 20.920}

Chile no tiene actualmente una gran industria de reciclaje, lo que se vincula con «los escasos incentivos para la reducción y reutilización de desechos» (Cepal, 2016: 25). Recién el 1 de junio de 2016 fue publicada en el Diario Oficial la Ley 20.920, que establece un marco para la gestión de residuos, la responsabilidad extendida del productor y fomento al reciclaje. En artículo 1 señala que tiene por objeto «disminuir la generación de residuos y fomentar su reutilización, reciclaje y otro tipo de valorización, a través de la instauración de la responsabilidad extendida del productor y otros instrumentos de gestión de residuos, con el fin de proteger la salud de las personas y el medio ambiente», y establece una serie de principios y un diseño institucional marco para la gestión de residuos.

La Ley se basa en una serie de reglamentos y actos complementarios (decretos, certificaciones y sistemas informáticos) para su implementación efectiva. El primero, el Reglamento de la Ley 20.920, describe el procedimiento para la dictación de los decretos supremos que fijan metas de recolección y valorización y otras obligaciones asociadas. Con procedimientos que incluyen consultas públicas, la dictación de los

4. Son rellenos sanitarios que se presentan como una solución factible en aquellos casos en que no se cuenta con equipo necesario para construir y operar un relleno sanitarios convencional. Se utiliza para poblaciones urbanas y rurales en general de menos de treinta mil habitantes. 
decretos de metas y valorización ha tardado más de lo esperado. A la fecha, se encuentran en tramitación solamente dos decretos para los productos prioritarios de neumáticos, ${ }^{5}$ y envases y embalajes. ${ }^{6}$

Si bien estimamos que la Ley 20.920 representa un avance en la gestión de residuos, su lenta implementación ha significado un atraso en esta materia. Hay que considerar que Chile es el segundo país de la Organización para la Cooperación y el Desarrollo Económicos (OCDE) que más basura envía a los rellenos sanitarios y vertederos (Cepal, 2016: 104).

Como señala el Informe Evaluaciones del Desempeño Ambiental: Chile 2016, «la plena aplicación de la ley contribuiría a reducir significativamente el volumen de desechos sólido descargados en los vertederos municipales, el correspondiente costo financiero para los gobiernos locales, y los riesgos ambientales y para la salud relacionados con el depósito de productos peligrosos en vertederos» (Cepal, 2016: 25) (énfasis nuestro), sin embargo, actualmente vemos muy lejana la dictación de todos los decretos de productos prioritarios.

\section{Normas generales, organismo sectorial y educación ambiental}

La inexistencia de una norma de orden general para la gestión de residuos sólidos ha generado un entramado normativo complejo a la hora de analizar la normativa aplicable. Por el contrario, y como veremos más adelante, hoy existen muchas normas con disposiciones respectos de los distintos tipos de residuos, lo que complejiza no solo la gestión institucional, sino también la particular, tanto de las industrias como de los ciudadanos.

Por otra parte, la inexistencia de un solo organismo sectorial a cargo de los residuos que abarque todas las competencias - tanto la gestión, como la fiscalización y eventualmente sanción- generará distorsiones institucionales al momento de la completa implementación de la Ley 20.920. Actualmente existen, respecto de la gestión de residuos, competencias tanto sanitarias como ambientales y económicas (seremis de Salud, municipalidades, la Superintendencia de Medio Ambiente a través de la fiscalización de las resoluciones de calificación ambiental, etcétera).

Pero el problema no es solo normativo o institucional. Un tercer factor que identificamos en nuestro análisis tiene que ver con educación ambiental. El Informe del estado del medio ambiente 2016 señala que efectivamente «la principal problemática

5. El anteproyecto fue dictado por medio de Resolución Exenta 0897 del Ministerio del Medio Ambiente, del 28 de septiembre de 2018. Durante la consulta pública, que se realizó entre el 4 de octubre y el 20 de noviembre de 2018, se recibieron 137 observaciones.

6. El anteproyecto fue dictado por medio de Resolución Exenta 0544 del Ministerio del Medio Ambiente, del 30 de mayo de 2019. El plazo de la consulta pública fue ampliado, hasta el día 13 de agosto de 2019. En la primera etapa se recibieron 624 observaciones. 
de los residuos del país está relacionada con la falta de reciclaje [lo cual] requiere un actuar decidido por el conjunto de la sociedad, con el fin de disminuir la cantidad de residuos que se disponen, así como promover la prevención y valorización de residuos» (SINIA, 2018: 328).

Es así como estos tres factores (normativo, institucional y educacional) se han conjugado para que hoy Chile aumente progresivamente la generación de desechos (30\% en los años 2000) y para que los vertederos sean el principal sitio de disposición final (Cepal, 2016: 25).

\section{Recuperación de suelos y valorización de residuos: Minería de rellenos sanitarios}

Esta sección pretende definir y conceptualizar los aspectos básicos de los distintos tipos de proyectos que es posible evaluar, con el objeto de recuperar las áreas que actualmente ocupan vertederos y rellenos sanitarios.

El diagnóstico respecto de la basura en Chile nos entrega datos abrumantes (tabla 1). Son 197 sitios catastrados, que fueron, son o serán utilizados para la acumulación de basura y residuos sólidos. Son fuentes infecciosas, de malos olores y que generan permanentes problemas de desigualdad al estar emplazados mayoritariamente en comunas de bajos recursos.

Tabla 1. Sitios de disposición de residuos a nivel nacional (Subdere, 2018: 14).

\begin{tabular}{lllllll|}
$\begin{array}{l}\text { Rellenos } \\
\text { sanitarios }\end{array}$ & $\begin{array}{l}\text { Relleno } \\
\text { manual }\end{array}$ & Vertederos & Basurales & Cerrados & Proyectos & Total \\
\hline 30 & 8 & 52 & 38 & 56 & 13 & 197 \\
\hline
\end{tabular}

Frente a esta realidad, creemos que es relevante comenzar a preguntarse qué hacer con todas aquellas instalaciones cerradas o que se encuentran próximas a su cierre. La recuperación de esos suelos parece una alternativa atractiva frente a la posibilidad de su abandono permanente.

Tradicionalmente, un suelo puede ser contaminado de diferentes formas: con metales pesados, lluvias ácidas, salinización, con elementos fitosanitarios, con residuos de explotaciones mineras o incluso con contaminantes orgánicos (Arraiza y otros, 2014: 10 y ss.). El suelo de un vertedero o de un relleno sanitario probablemente va a estar contaminado por uno o más de estos elementos, por los que probablemente será necesario utilizar más de una de las técnicas tradicionales de recuperación, a saber, la contención, confinamiento y descontaminación (Arraiza y otros, 2014: 22 y ss.).

En los últimos años, las investigaciones se han enfocado «en tratar de recuperar los suelos contaminados en vez de destruirlos» (Arraiza y otros, 2014: 22). De dichos estudios, actualmente surge la disponibilidad de «un amplio abanico de tecnologías 
de recuperación de suelos contaminados, algunas de aplicación habitual y otras todavía en fase experimental, diseñadas para aislar o destruir las sustancias contaminantes alterando su estructura química mediante procesos generalmente químicos, térmicos o biológicos» (Arraiza y otros, 2014: 22). De esta forma, la recuperación de suelos se vuelve una alternativa real en la actualidad.

Dada la posibilidad de recuperar suelos contaminados, es necesario plantearse qué hacer con todos los residuos acumulados en un vertedero, basural o relleno sanitario, para poder recuperar efectivamente los suelos que existen debajo. En este sentido, el landfill mining aparece como una atractiva alternativa que genera no solo la posibilidad de recuperar dichos suelos, sino que además obtener recursos a través de la valorización de los residuos ya dispuestos finalmente.

La Ley 20.920 define la valorización como el «conjunto de acciones cuyo objetivo es recuperar un residuo, uno o varios de los materiales que lo componen y/o el poder calorífico de los mismos. La valorización comprende la preparación para la reutilización, el reciclaje y la valorización energética». Sin embargo, nuestra normativa nacional no establece mayor regulación al respecto. Como veremos más adelante, la valorización de residuos es parte de los proyectos de minería de vertederos.

La expresión inglesa landfill mining se forma combinando los conceptos de vertedero y minería, y podría describirse como "un proceso para extraer minerales u otros recursos naturales sólidos de los materiales de desecho que previamente se eliminaron enterrándolos en el suelo» (Krook, Svensson y Eklund, 2012: 513).?

La minería de los vertederos o de los rellenos sanitarios puede ser usada tanto para remediar un mal diseño o una mala operación de los rellenos sanitarios, como para actualizar rellenos sanitarios que no cumplen con los requisitos ambientales y especificaciones sanitarias, ${ }^{8}$ o para derechamente obtener recursos a partir de su explotación.

De acuerdo con la World Resource Foundation, el procedimiento para implementar la minería de rellenos sanitarios consiste principalmente en excavar el material depositado, procesarlo de manera tal que el material objetivo pueda separarse de la masa excavada y luego volver a procesarlo para obtener el producto. ${ }^{9}$ La excavación se realiza utilizando técnicas similares a las de la minería a cielo abierto. Luego, el procesamiento comienza con la segregación de la masa excavada, la que se descarga en una pantalla gruesa que tiene como fin eliminar los desechos no procesables de gran tamaño. El material de la fracción restante se retira y se expone a un imán para

7. En el original: «A process for extracting minerals or other solid natural resources from waste materials that previously have been disposed of by burying them in the ground».

8. World Resource Foundation, «Landfill Mining», disponible en http://www.enviroalternatives.com/ landfill.html.

9. World Resource Foundation, «Landfill Mining». 
recuperar metales ferrosos. La fracción no ferrosa se procesa a través de un clasificador de aire que separa los materiales orgánicos ligeros de los orgánicos pesados. De esta forma, es posible obtener tanto productos minerales como energía derivada del procesamiento de los materiales orgánicos.

La literatura ha identificado las principales funciones de la minería de rellenos sanitarios (Hogland, Marques y Thorneby, 1997: 341):

- Reducción en el área del vertedero, terminación o cierre del vertedero.

- Descontaminación total del suelo.

- Recuperación de materiales y/o construcción de un nuevo relleno sanitario.

- Actualización e instalación de un revestimiento inferior del vertedero para proteger el suelo.

- Reducción de volumen/continuación de la operación del vertedero en el sitio existente.

- Recuperación y reutilización de materiales, como: i) suelo como material de cobertura; ii) recuperación de material para producción de energía y subproductos metálicos.

La ventaja que ofrece la minería de rellenos sanitarios es que, a través de un mismo proceso, se puede obtener productos, alargar la vida útil de un vertedero, obtener energía, o recuperar los suelos. Las posibilidades y ventajas de incorporar este tipo de tecnologías se traduce en las múltiples combinaciones de sus beneficios.

La primera vez que se utilizó el concepto de landfill mining fue en Israel, en el año 1953 «como una forma de obtener fertilizantes para huertos, pero esta siguió siendo la única iniciativa reportada por varias décadas» (Krook, Svensson y Eklund, 2012: 514). ${ }^{10}$ Durante los años noventa, en Estados Unidos - y por efecto de la regulación a los vertederos - ${ }^{11}$ "obtener permisos para desarrollar nuevos vertederos era cada vez más difícil, principalmente debido a la fuerte oposición pública» (Krook, Svensson y Eklund, 2012: 514). ${ }^{12}$ Por lo anterior, los dueños comenzaron a buscar estrategias para reducir o posponer los costos relacionados con el cierre final, aumentando la vida útil de los vertederos. La «excavación, procesamiento, tratamiento y recuperación de los materiales depositados emergieron como una estrategia prometedora para resolver

10. Traducción de las autoras.

11. De acuerdo con los autores, las regulaciones de vertederos de la época obligaron a muchos a cerrar, implicando también requisitos más estrictos sobre el cierre final y la posterior gestión y monitoreo a largo plazo de contaminantes (Krook, Svensson y Eklund 2012, 513-520)

12. Traducción de las autoras. 
la creciente disminución de capacidad» (Krook, Svensson y Eklund, 2012: 514). ${ }^{13}$ Sin embargo, el objetivo principal era el aumento de capacidad del sitio de disposición y no la obtención de recursos a partir del mismo.

En los Países Bajos se han impulsado algunos proyectos de minería de vertederos. Los principales motivos para el desarrollo de estos proyectos en este país se han relacionado con el uso del suelo (el desarrollo de un área industrial en la ubicación del vertedero) y para evitar la contaminación del entorno. Solo en uno de los casos fue por la obtención de un producto final propiamente tal (Van der Zee, Achterkamp y Visser, 2004: 5). Actualmente, existen varios países realizando estudios para evaluar la posibilidad de implementar el landfill mining en sus vertederos. Escocia (WRAP, 2013) e India (Dubey, Chakrabarti y Pandit, 2016: 319-327) tienen importantes análisis costo-beneficio al respecto. Nuestro objetivo ahora es intentar dilucidar si normativamente es posible, o no, incorporar estas nuevas tecnologías a nuestro país y analizar el impacto que implicaría en el cambio climático.

\section{Marco normativo chileno: residuos sólidos}

En Chile no existe una ley o normativa de orden general que regule la gestión de los residuos sólidos. Lo que hay es una gran cantidad de normas referidas a distintas materias o actividades que contienen disposiciones asociadas al manejo, gestión, almacenamiento, transporte o disposición final de los residuos sólidos de diferentes clases. En esa línea, existen distintos organismos sectoriales con competencias en materia de residuos: la autoridad sanitaria, quien otorga autorizaciones y fiscaliza; las municipalidades, encargadas de la gestión de los residuos sólidos domiciliarios; la Superintendencia del Medio Ambiente, que fiscaliza aquellos casos en que el proyecto cuenta con una resolución de calificación ambiental.

En ese sentido, podemos encontrar disposiciones que regulan distintas actividades en relación con los residuos sólidos en los siguientes cuerpos normativos:

- Ley 20.920, que establece un marco para la gestión de residuos, la responsabilidad extendida del productor y el fomento al reciclaje, del Ministerio del Medio Ambiente. Esta ley fue publicada el 1 de junio de 2016 y, como señalamos antes, tiene como objeto «disminuir la generación de residuos y fomentar su reutilización, reciclaje y otro tipo de valorización, a través de la instauración de la responsabilidad extendida del productor y otros instrumentos de gestión de residuos, con el fin de proteger la salud de las personas y el medio ambiente» (artículo 1).

- Decreto con Fuerza de Ley 725 de 1967, Código Sanitario, del Ministerio de Salud Pública. Esta norma regula todas las cuestiones relacionadas con el fo-

13. Traducción de las autoras. 
mento, protección y recuperación de la salud de los habitantes de la República. En ese marco, establece como una de las atribuciones y obligaciones de las municipalidades la de «recolectar, transportar y eliminar por métodos adecuados, a juicio del Servicio Nacional de Salud, las basuras, residuos y desperdicios que se depositen o produzcan en la vía urbana», de acuerdo con lo establecido en el artículo 11 letra b) de la norma. Asimismo, en el párrafo III del Título II del Libro III, referido a los desperdicios y basuras, la norma exige, específicamente en los artículos 79 y 89, autorización sanitaria para la «construcción, reparación, modificación y ampliación de cualquier planta de tratamiento de basuras y desperdicios de cualquier clase», así como para «el funcionamiento de todo lugar destinado a la acumulación, selección, industrialización, comercio o disposición final de basuras y desperdicios de cualquier clase».

- Decreto con Fuerza de Ley 1, que fija el texto refundido, coordinado y sistematizado de la Ley 18.695, Orgánica Constitucional de Municipalidades, del Ministerio del Interior. Esta norma establece, en su artículo 3 letra f), el aseo y ornato de la comuna como función privativa de las municipalidades; a estas le corresponde la recolección, transporte y/o disposición final de los residuos domiciliarios. Asimismo, en el artículo 25 le atribuye el servicio de extracción de la basura a la unidad encargada de la función de medio ambiente, aseo y ornato.

- Decreto Ley 3.063 de 1979, Ley de Rentas Municipales, del Ministerio del Interior. Esta norma, en su artículo 6, se refiere al cobro del servicio municipal de extracción de residuos sólidos domiciliarios, estableciendo la posibilidad de que este cobro sea diferenciado, utilizando para ello diversos criterios, como programas ambientales, incluyendo reciclaje, frecuencia o volúmenes de extracción, o las condiciones de accesibilidad.

- Ley 19.300, que aprueba Ley sobre Bases Generales del Medio Ambiente, del Ministerio Secretaría General de la Presidencia. Esta ley, publicada en marzo de 1994 y modificada por la Ley 20.417 el año 2010, exige la evaluación ambiental de una serie de proyectos en su artículo 10, entre ellos los establecidos en los literales i) y o), consistentes en proyectos de desarrollo minero, incluidos los de disposición de residuos y estériles, así como los proyectos de saneamiento ambiental, como plantas de tratamiento de residuos sólidos de origen domiciliario, rellenos sanitarios, y sistemas de tratamiento y disposición de residuos industriales líquidos o sólidos, respectivamente. Asimismo, entre las funciones del Ministerio del Medio Ambiente, el artículo 70 letra g) establece la de «proponer políticas y formular normas, planes y programas en materia de residuos y suelos contaminados». 
- Ley 20.879, que sanciona el transporte de desechos hacia vertederos clandestinos, del Ministerio de Transportes y Telecomunicaciones. Ley publicada en noviembre de 2015, y que modifica la Ley del Tránsito, introduciendo tres nuevos artículos (192 bis, 192 ter y 192 quáter) referidos a las sanciones asociadas al transporte, traslado o depósito de basuras, desechos o residuos de cualquier tipo, hacia o en la vía pública, sitios eriazos, en vertederos o depósitos clandestinos o ilegales, o en los bienes nacionales de uso público; y al transporte y retiro de escombros sin contemplar las medidas necesarias para evitar la dispersión de materiales o polvo durante su traslado.

- Decreto Supremo 40 de 2012, que aprueba el Reglamento del Sistema de Evaluación de Impacto Ambiental, del Ministerio del Medio Ambiente. Este reglamento tiene como objeto establecer las disposiciones por las cuales se regirá el Sistema de Evaluación de Impacto Ambiental (SEIA) y la Participación de la Comunidad en el proceso de evaluación de impacto ambiental. Con ese propósito, detalla en su artículo 3 cada uno de los proyectos que de acuerdo a la Ley 19.300 deben ser sometidos al SEIA, entre ellos los establecidos en los literales i) y o), ya identificados.

- Decreto Supremo 594 de 1999, que establece condiciones sanitarias y ambientales básicas en los lugares de trabajo, del Ministerio de Salud. Esta norma trata, en el párrafo III del título II, la disposición de residuos industriales líquidos y sólidos, exigiendo autorización sanitaria para su almacenamiento, transporte, tratamiento y disposición final. Al respecto señala en el artículo 18 que «la acumulación, tratamiento y disposición final de residuos industriales dentro del predio industrial, local o lugar de trabajo, deberá contar con la autorización sanitaria». Por su parte, el artículo 19 dispone que «las empresas que realicen el tratamiento o disposición final de sus residuos industriales fuera del predio, sea directamente o a través de la contratación de terceros, deberán contar con autorización sanitaria, previo al inicio de tales actividades». Luego, en el artículo 20 señala un listado de residuos que serán entendidos como peligrosos para efectos del Reglamento.

- Decreto Supremo 148 de 2004, que establece el Reglamento Sanitario sobre el Manejo de Residuos Peligrosos, del Ministerio de Salud. Este reglamento regula «las condiciones sanitarias y de seguridad mínimas a que deberá someterse la generación, tenencia, almacenamiento, transporte, tratamiento, reúso, reciclaje, disposición final y otras formas de eliminación de los residuos peligrosos».

- Decreto Supremo 189 de 2008, que aprueba el reglamento sobre condiciones sanitarias y de seguridad básicas en los rellenos sanitarios, del Ministerio de 
Salud. La presente norma, con el objeto de regular las condiciones sanitarias y de seguridad básicas de los rellenos sanitarios, dispone en su artículo 5 que «todo relleno sanitario deberá contar con un proyecto de ingeniería aprobado por la autoridad sanitaria, el que deberá ser elaborado por un profesional idóneo. En aquellos casos en que previamente corresponda ingresar el proyecto al Sistema de Evaluación de Impacto Ambiental, la autoridad sanitaria otorgará dicha aprobación una vez que, habiendo sido incorporados las exigencias contenidas en la respectiva Resolución de Calificación Ambiental, se constate el cabal cumplimiento de las disposiciones reglamentarias, de orden sanitario y de seguridad». Asimismo, establece los contenidos mínimos del proyecto de ingeniería.

- Decreto supremo 6 de 2009, que aprueba el reglamento sobre manejo de residuos generados en establecimientos de atención de salud, del Ministerio de Salud. La presente norma, clasifica los residuos generados en establecimientos de atención de salud en cuatro categorías para regular su manejo, y entrega una definición para efectos del reglamento: residuos peligrosos, residuos radioactivos de baja intensidad, residuos especiales y residuos sólidos asimilables a domiciliarios.

- Decreto Supremo 3 de 2012, que aprueba el reglamento para el manejo de lodos provenientes de plantas de tratamiento de efluentes de la industria procesadora de frutas y hortalizas, del Ministerio del Medio Ambiente. La presente norma, de acuerdo con su artículo 1, «establece las exigencias sanitarias mínimas para el manejo, restricciones, requisitos y condiciones técnicas, para la aplicación de lodos en determinados suelos», definiendo lodo, para efectos de este reglamento, como «residuo semisólido generado en plantas de tratamiento de efluentes líquidos de la industria procesadora de frutas y verduras».

- Decreto Supremo 4 de 2009, que establece el reglamento para el manejo de lodos generados en plantas de tratamiento de aguas servidas, del Ministerio Secretaría General de la Presidencia. Este reglamento, de acuerdo con su artículo 1, «establece la clasificación sanitaria de los lodos y las exigencias sanitarias mínimas para su manejo, además de las restricciones, requisitos y condiciones técnicas para la aplicación de lodos en determinados suelos», definiendo lodos como «residuos semisólidos que hayan sido generados en plantas de tratamiento de aguas servidas».

- Decreto Supremo 2 de 2010, que regula autorización de movimientos transfronterizos de residuos peligrosos consistentes en baterías de plomo usadas, del Ministerio de Salud. Esta norma establece la prohibición de «movimiento transfronterizo de baterías de plomo usadas, desde Chile a terceros países, en 
tanto existan en el país instalaciones con capacidad para procesar estos residuos peligrosos».

- Decreto Supremo 29 de 2013, que establece la norma de emisión para la incineración, coincineración y coprocesamiento, del Ministerio del Medio Ambiente. La norma regula las emisiones de «las instalaciones de incineración, las instalaciones de coincineración que correspondan a hornos rotatorios de cal o a instalaciones forestales y para las instalaciones de coprocesamiento que correspondan a hornos de cemento, que utilicen combustibles distintos a los tradicionales». Asimismo, en su artículo 1 establece que «su objetivo es prevenir los efectos negativos sobre la salud de la población y los recursos naturales, derivados de las emisiones tóxicas provenientes de los procesos de incineración, coprocesamiento y coincineración regulados por este decreto».

- Decreto Supremo 1 de 2013, que aprueba el Reglamento del Registro de Emisiones y Transferencias de Contaminantes (RETC), del Ministerio del Medio Ambiente. El artículo 1 de la norma establece que el RETC «es una base de datos accesible al público, destinada a capturar, recopilar, sistematizar, conservar, analizar y difundir la información sobre emisiones, residuos y transferencias de contaminantes potencialmente dañinos para la salud y el medio ambiente que son emitidos al entorno, generados en actividades industriales o no industriales o transferidos para su valorización o eliminación». El RETC «dispondrá de manera sistematizada, por fuente o agrupación de fuentes, la naturaleza, caudal y concentración de emisiones de contaminantes que sean objeto de una norma de emisión. Además, el registro contemplará la declaración o estimación de emisiones, residuos y transferencias de aquellos contaminantes que no se encuentran regulados en una norma de emisión, plan de descontaminación, $\mathrm{u}$ otra regulación vigente, cuando se trate de emisiones que corresponden a fuentes difusas, o que se estiman debido a que se encuentran en convenios internacionales suscritos por Chile. Las estimaciones las realizará el Ministerio del Medio Ambiente mediante la información que entreguen los diferentes órganos de la Administración del Estado». La disposición añade que «el registro contemplará la cantidad, naturaleza, características, origen, destino y la gestión de los residuos generados por los establecimientos, de conformidad a lo dispuesto en el presente reglamento y, en particular, de los residuos de productos prioritarios».

Nos parece que es fundamental $-\mathrm{y}$ urgente - sistematizar la normativa asociada a residuos en nuestra legislación, idealmente a través de una ley marco de residuos que establezca normas claras para todo tipo de residuos, respecto de su gestión, almacenamiento, transporte, reutilización, valorización, reciclaje y disposición final, 
esperando que esta última —la disposición final— sea dejada solo en caso que efectivamente no exista otra alternativa, con el objeto de avanzar hacia una reducción efectiva de los rellenos sanitarios y vertederos.

\section{Conceptos básicos en torno a los residuos sólidos}

Nuestra legislación define residuo en al menos tres cuerpos normativos. En su artículo 3, el Reglamento Sanitario sobre el Manejo de Residuos Peligrosos establece que residuo o desecho es toda «sustancia, elemento u objeto que el generador elimina, se propone eliminar o está obligado a eliminar». El Decreto Supremo 189 de 2008 establece que residuo, basura, desecho o desperdicio son las «sustancias, elementos u objetos cuyo generador elimina, se propone eliminar o está obligado a eliminar». Por su parte, la reciente Ley 20.920 define de manera genérica el concepto de residuo, en el artículo 3 numeral 25), señalando que este es toda «sustancia u objeto que su generador desecha o tiene la intención u obligación de desechar de acuerdo a la normativa vigente». El Reglamento del RETC en su artículo 3 letra n) lo define del mismo modo.

Si bien no hay una definición normativa de residuo sólido en general, la Ley de Rentas Municipales señala en su artículo 6 que se considerarán residuos sólidos domiciliarios a las «basuras de carácter doméstico generadas en viviendas y en toda otra fuente cuyos residuos presenten composiciones similares a los de las viviendas». El Decreto Supremo 189 establece en su artículo 4 que residuos sólidos domiciliarios son los «residuos sólidos, basuras, desechos o desperdicios generados en viviendas y en establecimientos tales como edificios habitacionales, locales comerciales, locales de expendio de alimentos, hoteles, establecimientos educacionales y cárceles», mientras que los residuos sólidos asimilables son los «residuos sólidos, basuras, desechos o desperdicios generados en procesos industriales u otras actividades, que no son considerados residuos peligrosos de acuerdo a la reglamentación sanitaria vigente y que, además, por su cantidad composición y características físicas, químicas y bacteriológicas, pueden ser dispuestos en un relleno sanitario sin interferir con su normal operación». Por su parte, el Decreto Supremo 594 de 1999 dispone en su artículo 18 que se entenderá por residuo industrial «todo aquel residuo sólido o líquido, o combinaciones de estos, provenientes de los procesos industriales y que, por sus características físicas, químicas o microbiológicas no puedan asimilarse a los residuos domésticos». Por último, el Reglamento Sanitario sobre el Manejo de Residuos Peligrosos dispone en su artículo 3 que residuo peligroso es todo «residuo o mezcla de residuos que presenta riesgo para la salud pública y/o efectos adversos al medio ambiente, ya sea directamente o debido a su manejo actual o previsto, como consecuencia de presentar algunas de las características señaladas en el artículo 11».

La eliminación ha sido entendida tanto por el Reglamento del RETC, en su artículo literal c), como en la Ley 20.920 , en su artículo 3 numeral 8), como «todo pro- 
cedimiento cuyo objetivo es disponer en forma definitiva o destruir un residuo en instalaciones autorizadas».

El relleno sanitario, como lugar de disposición definitiva, es definido en el artículo 4 del Decreto Supremo 189 como la «instalación de eliminación de residuos sólidos en la cual se disponen residuos sólidos domiciliarios y asimilables, diseñada, construida y operada para minimizar molestias y riesgos para la salud y la seguridad de la población y daños para el medio ambiente, en la cual las basuras son compactadas en capas al mínimo volumen practicable y son cubiertas diariamente, cumpliendo con las disposiciones del presente reglamento».

En el mismo artículo también define el biogás como la «mezcla de gases generada por la descomposición anaeróbica de la materia orgánica putrescible de los residuos que se depositan en un relleno sanitario y que consiste mayoritariamente en metano, dióxido de carbono, vapor de agua y, en mucho menor medida, de gases tales como sulfuro de hidrógeno e hidrógeno».

En ninguna de las normas vigentes en nuestro país asociadas a residuos — tal vez solo excluyendo a Ley 20.920 - podemos encontrar el establecimiento de principios claros que inspiren la regulación, y que tiendan inequívocamente a reducir el impacto climático que la basura genera. Es de suma relevancia actualizar estas normas a la luz de los desafiantes objetivos que el país se ha planteado respecto a la disminución de los gases de efecto invernadero: bajar progresivamente hasta un nivel de $95 \mathrm{Mt}$ $\mathrm{CO}_{2}$ en 2030 y carbono neutralidad en 2050, según la última actualización de la Contribución Nacional al Acuerdo Climático de París 2015.

\section{Tipos de residuos sólidos en nuestro ordenamiento}

De las definiciones entregadas por los distintos cuerpos normativos vigentes en nuestro ordenamiento, y de lo dispuesto por el Ministerio del Medio Ambiente a través del sitio web del Sistema Nacional de Información Ambiental, ${ }^{14}$ podemos concluir que los residuos sólidos pueden clasificarse según su origen y según sus características.

Según su origen:

- Residuos sólidos municipales: Son aquellos residuos sólidos domiciliarios y residuos asimilables a los anteriores generados en el sector servicios y pequeñas industrias. También se consideran residuos municipales a los derivados del aseo de vías públicas, áreas verdes y playas.

- Residuos industriales: Residuo resultante de los procesos de fabricación, transformación, utilización, consumo, limpieza y mantenimiento, generados por

14. Sistema Nacional de Información Ambiental (SINIA), Ministerio de Medio Ambiente, «Residuos», disponible en https://sinia.mma.gob.cl/temas-ambientales/residuos/. 
la actividad industrial. Corresponden a residuos que, por sus características físicas, químicas o microbiológicas, no pueden asimilarse a los residuos domésticos.

Según sus características:

- Residuo peligroso: Residuo o mezcla de residuos que presenta riesgo para la salud pública y/o efectos adversos al medio ambiente, ya sea directamente o debido a su manejo actual o previsto.

- Residuo no peligroso: Residuo que no presenta riesgo para la salud pública ni efectos adversos al medio ambiente.

- Residuo inerte: Es un residuo no peligroso que no experimenta variaciones físicas, químicas o biológicas significativas, no es soluble, ni combustible, ni reacciona física o químicamente, ni de ninguna otra manera. No es biodegradable y tampoco afecta negativamente a otras materias con las cuales entra en contacto.

\section{Evaluación ambiental de los proyectos de recuperación de suelos}

Así como no existe una ley marco para la gestión de residuos en Chile, tampoco existe un marco regulatorio para la protección y calidad del uso de los suelos..$^{15} \mathrm{No}$ tenemos una norma que nos indique con claridad cuándo se entiende que un suelo está contaminado, qué acciones se deben tomar para recuperarlo, ni una metodología estandarizada para el análisis de suelo.

De acuerdo con lo señalado por el Ministerio del Medio Ambiente en su Cuarto reporte del estado del medio ambiente (SINIA, 2018: 192), específicamente en el capítulo relacionado al suelo, el últimos esfuerzo en esta materia es el análisis hecho en 2011 sobre la posibilidad de normar el componente suelo a través de un instrumento vinculante, para lo cual mandató la realización del estudio «Preparación de antecedentes para la elaboración de la norma de calidad primaria de suelos». Como consecuencia de las recomendaciones de ese estudio, el año 2013 se publicó la Guía metodológica para la gestión de suelos con potencial presencia de contaminantes, aprobada mediante la Resolución Exenta 406, la que expone los principales procedimientos involucrados

15. Existen distintos cuerpos normativos que regulan el suelo: la Ley 20.551, sobre cierre de faenas mineras; la Ley 19.300 sobre Bases Generales de Medio Ambiente; la Ley 20.412, sobre incentivos agrícolas a suelos degradados; el Decreto con Fuerza de Ley 235, que establece un sistema de incentivos para la recuperación de suelos degradados; el Decreto 248, que aprueba el reglamento para la aprobación de proyectos de diseño, construcción, operación y cierre de los depósitos de relaves; y el Decreto Ley 3.557, que establece disposiciones sobre protección agrícola. 
en la gestión de suelos con potencial presencia de contaminantes. Pero este esfuerzo es claramente insuficiente.

La degradación del suelo se define como un «cambio en la salud del suelo resultando en una disminución de la capacidad del ecosistema para producir bienes o prestar servicios para sus beneficiarios. Los suelos degradados contienen un estado de salud que no puede proporcionar los bienes y servicios normales del suelo en cuestión en su ecosistema» (BCN, 2019). Esta degradación del suelo es fundamentalmente producida por el hombre a través de actividades forestales, de agricultura, ganaderas, o bien indirectamente a través de actividades industriales, mineras, de transporte y por la disposición final de residuos. De acuerdo a los datos levantados en el mencionado Cuarto reporte del estado del medio ambiente, un $24,25 \%$ de los sitios potencialmente contaminados a nivel nacional tienen su origen en la disposición de residuos (SINIA, 2018: 200).

Como señalamos, la Ley 19.300 establece en su artículo 10 un listado de todos aquellos proyectos que deben ser evaluados ambientalmente, los que luego el RSEIA en su artículo 3 se encarga de detallar y definir. En el literal o) de ambos artículos se señala que deben evaluarse ambientalmente los "proyectos de saneamiento ambiental, tales como sistemas de alcantarillado y agua potable, plantas de tratamiento de agua o de residuos sólidos de origen domiciliario, rellenos sanitarios, emisarios submarinos, sistemas de tratamiento y disposición de residuos industriales líquidos o sólidos». Luego, el artículo 3 del RSEIA detalla qué se entenderá por proyectos de saneamiento ambiental. Especial atención nos merecen los siguientes literales:

Se entenderá por proyectos de saneamiento ambiental al conjunto de obras, servicios, técnicas, dispositivos o piezas que correspondan a: [...]

0.5. Plantas de tratamiento y/o disposición de residuos sólidos de origen domiciliario, rellenos sanitarios, estaciones de transferencia y centros de acopio y clasificación que atiendan a una población igual o mayor a cinco mil (5.000) habitantes. [...]

o.8. Sistemas de tratamiento, disposición y/o eliminación de residuos industriales sólidos con una capacidad igual o mayor a treinta toneladas día (30 t/día) de tratamiento o igual o superior a cincuenta toneladas (5o t) de disposición.

o.9. Sistemas de tratamiento, disposición y/o eliminación de residuos peligrosos con una capacidad de veinticinco kilos día ( $25 \mathrm{~kg} /$ día) para aquellos que estén dentro de la categoría de «tóxicos agudos» según DS 148/2003 Ministerio de Salud; y de mil kilos día (1000 kg/día) para otros residuos peligrosos.

o.10 Sistemas de tratamiento, disposición y/o eliminación de residuos especiales provenientes de establecimientos de salud, con capacidad mayor o igual a doscientos cincuenta kilogramos diarios (250 kg/día).

o.11 Reparación o recuperación de áreas que contengan contaminantes, que abarquen, en conjunto, una superficie igual o mayor a diez mil metros cuadrados (10.000 
$\mathrm{m}^{2}$ ), salvo que se trate de medidas que formen parte de una propuesta de plan de reparación a que se refiere el artículo 43 de la Ley Orgánica de la Superintendencia del Medio Ambiente, cuyo texto fue fijado por el artículo segundo de la Ley 20.417, caso en el cual se aplicará lo dispuesto en dicha disposición y en su Reglamento.

De lo anterior, podemos concluir que en nuestro país deben ser evaluados ambientalmente todos aquellos proyectos que contemplen la disposición final de residuos sobre los límites establecidos, así como aquellos que contemplen la reparación o recuperación de suelos contaminados, que abarquen, en conjunto, una superficie igual o mayor a una hectárea. Por lo tanto, a falta de normativa asociada al componente suelo, y a la recuperación de suelos contaminados, el Sistema de Evaluación de Impacto Ambiental ha debido hacerse cargo de establecer medidas para evitar, minimizar, compensar o reparar impactos al suelo generados por proyectos, pero solo respecto de aquellos que requieren ingresar al dicho sistema.

Revisados los proyectos sometidos al SEIA por el literal o.11 antes identificado, ${ }^{16}$ podemos señalar que, de 143 ingresos al sistema, 87 proyectos han sido aprobados desde que existe. De este universo, 60 corresponden a proyectos asociados a planes de cierre o a estudios de cierre de vertederos o rellenos sanitarios, los que tienen en general como finalidad el implementar el cierre progresivo y sellado del sitio de disposición final, permitiendo minimizar los riesgos sanitarios y ambientales asociados al manejo de los residuos sólidos. De los proyectos aprobados a la fecha, solo dos tienen por objeto la recuperación y habilitación del terreno mediante un saneamiento del suelo intervenido a través de la remoción del material residual existente: uno abarcando una superficie de 3,44 hectáreas y otro, una superficie de 15,8 hectáreas. ${ }^{17}$

A nuestro parecer, el literal de ingreso no se ha utilizado correctamente a la fecha, ya que en su gran mayoría no se han evaluado proyectos de reparación o recuperación de suelos, sino planes de cierre de sitios que buscan solo minimizar mayores efectos ambientales y para la salud. Queda pendiente la tarea de evaluar si este literal debe ser ampliado para incorporar expresamente los planes de cierre de sitios de disposición final de residuos sólidos, o bien si lo que buscamos es efectivamente recuperar los suelos contaminados.

Dicho todo lo anterior, podemos concluir que nuestro ordenamiento sí contempla la evaluación de proyectos de recuperación de suelos en la que podría enmarcarse perfectamente un proyecto de landfill mining.

16. Análisis realizado a partir de la información del e-SEIA, al 5 de octubre de 2020.

17. Nos referimos a los proyectos «Recuperación departamental» (RCA 223/2020) y «Saneamiento del terreno Las Salinas» (RCA 24/2020). 


\section{La importancia del suelo en la contribución al cambio climático y los objetivos del Protocolo de Kioto}

No solo la degradación de los desechos orgánicos en vertederos y rellenos sanitarios genera emisiones de metano que a largo plazo contribuyen al calentamiento global, sino que también - especialmente los más antiguos- son fuente de contaminación local al producir lixiviación de sustancias peligrosas (Krook, Svensson y Eklund, 2012: 513) y, como consecuencia, la pérdida del suelo debajo de los vertederos genera importantes efectos a nivel climático.

El crecimiento económico y demográfico de nuestro país ha aumentado exponencialmente la producción de gases de efecto invernadero, los que son considerados la principal causa del calentamiento global y el cambio climático, ya que la degradación y pérdida de suelos conduce a la eliminación de uno de los principales sumideros de $\mathrm{CO}_{2}$ a nivel mundial. Como ya se adelantó, «el suelo ejerce su labor protectora a través de su poder de amortiguación o capacidad natural de depuración de la contaminación» (Ortiz Bernard y otros, 2007: 10).

En 2005, el Protocolo de Kioto reconoció la importancia de los suelos, señalando que las emisiones netas de carbono podrían ser reducidas a través de su acumulación en sumideros terrestres, pues los suelos son «uno de los principales sumideros de carbono de la naturaleza [...] con potencial para incrementar la retención de $\mathrm{CO}_{2} »$ (Ortiz Bernard y otros, 2007: 19). Sin embargo, Chile no contempla compromisos específicos en materia de recuperación de suelos. Los objetivos que persigue el país dicen relación, más bien, con energía y reforestación.

En cualquier caso, hay que tener muy presente que el poder de amortiguación de un suelo no es ilimitado, y cuando se rebasa, el suelo deja de ser eficaz como sumidero de la contaminación, llegando incluso a invertirse el proceso y a convertirse en una fuente de contaminación para los organismos del suelo y para el medio circundante (Ortiz Bernard y otros, 2007: 11). De ahí que sea tan importante, para la protección de este recurso, evaluar e incentivar el desarrollo y la innovación respecto de las técnicas de recuperación de suelos.

El problema es que, si bien el impacto final en la recuperación de suelos resulta positivo para el cambio climático y para la disminución de gases de efecto invernadero, el proceso de recuperación tiene efectos tanto positivos como negativos que es necesario evaluar.

Respecto del landfill mining, los análisis que se han dedicado a realizar estudios de impacto, se han enfocado en el análisis costo-beneficio de su implementación y han arrojado los resultados que se sintetizan en la tabla $2 .^{18}$

18. Para otros análisis costo-beneficio, véase Dubey, Chakrabarti y Pandit (2016: 319-327). 
Tabla 2. Costos y beneficios del landfill mining

Beneficios
Cumento de la capacidad del vertedero
Cierre de vertedero
Atención y seguimiento posteriores al cierre
Compra de capacidad adicional o sistemas sofisticados
Ingresos de:
Materiales reciclables y reutilizables
Residuos combustibles vendidos como combustible
Suelo recuperado utilizado como cubierta
materiales, vendidos como relleno de construcción, vendido para
otros usos
Responsabilidad por la remediación de áreas adyacentes
Valor de la tierra de sitios recuperados para otros usos

\section{Costos}

Gastos incurridos en la planificación del proyecto:

Preparación del sitio

Alquiler o compra de equipos

Alquiler o compra de equipos de seguridad

Construcción o expansión de instalaciones de manejo de materiales

Costos operativos:

Trabajo

Equipo de combustible y mantenimiento

Relleno de tierras de residuos no recuperados

Administrativo y regulatorio

Gastos de cumplimiento

Formación de trabajadores en seguridad y procedimientos.

Costos de transporte

Fuente: (Van der Zee, Achterkamp y Visser, 2004: 10)

Un estudio realizado en China (Zhou y otros, 2015) propone otros criterios para el análisis costo-beneficios del landfill mining (tabla 3), y que nos parecen especialmente relevantes dado que los escenarios evaluados van más allá de los costos operacionales de la tabla 2.

Tabla 3. Costos y beneficios de landfill mining según Zhou y otros (2015)

\begin{tabular}{|c|c|c|c|}
\hline Costos & & Beneficios & \\
\hline Capital & $\begin{array}{l}\text { Preparación del sitio } \\
\text { Maquinaria para excavaciones } \\
\text { Maquinaria para clasificación de } \\
\text { residuos y procesamiento } \\
\text { Maquinaria para manipulación de } \\
\text { materiales }\end{array}$ & Suelos & Obtención de suelos para desarrollo urbano \\
\hline \multirow{3}{*}{$\begin{array}{l}\text { Costos } \\
\text { operacionales }\end{array}$} & Estudios pre operacionales & $\begin{array}{l}\text { Materiales } \\
\text { recuperados }\end{array}$ & $\begin{array}{l}\text { Fertilizantes } \\
\text { Materiales de construcción } \\
\text { Metales reciclados }\end{array}$ \\
\hline & Transporte de material & Energía & $\begin{array}{l}\text { Combustible generada a través de la basura plástica } \\
\text { Energía producto de la valorización de residuos y otros } \\
\text { combustibles }\end{array}$ \\
\hline & Disposición final de residuos & Post cierre & $\begin{array}{l}\text { Evitar emisiones de gases de los vertederos } \\
\text { Tratamiento y cuidado de los lixiviados }\end{array}$ \\
\hline
\end{tabular}


Sin embargo, creemos que el análisis costo-beneficio no es el único que hay que tener en cuenta a la hora de evaluar alternativas de remediación de pasivos ambientales. Los estudios deben incluir análisis tanto económicos como técnicos, regulatorios, ambientales y sociales (WRAP, 2013). El análisis costo-efectividad también es relevante a la hora de evaluar proyectos de saneamiento de pasivos ambientales. Además, es necesario incorporar las variables climáticas y el análisis de las emisiones de gases de efecto invernadero para lograr evaluar si, efectivamente, la minería de rellenos sanitarios constituye una alternativa climáticamente viable a la hora de recuperar suelos.

Estudios recientes realizados en Sao Paulo, Brasil, han arrojado resultados positivos respecto del análisis de la experiencia comparada en la implementación de esta tecnología: se ha indicado que el landfill mining es significativamente útil para el cumplimiento de metas de desarrollo sustentable: funciona como una herramienta de protección ambiental de las aguas y de los residuos, como elemento de inclusión social y reducción de pobreza e inequidad y como forma de recuperación de suelos que pueden ser recuperados eficientemente para su posterior urbanización (Calderón y otros, 2019).

Aun cuando no se tengan estudios y datos respecto de la implementación de las diferentes técnicas de recuperación de suelos en Chile, es importante comenzar a discutir acerca de la posibilidad de desarrollar este tipo de proyectos. Entender la importancia que tienen los suelos y el impacto que genera la basura y su acumulación en vertederos y rellenos sanitarios nos obliga a pensar que, al menos, la industria debiese comenzar a investigar respecto de sus distintas alternativas de recuperación. Y que nosotros, como ciudadanos, también tenemos el deber de tomar conciencia respecto de nuestros propios residuos y su impacto climático.

\section{Conclusiones}

Los rellenos sanitarios y vertederos aparecieron en un primer momento como una solución inmediata a un problema sanitario asociado a la vida urbana, pero con el crecimiento de la ciudad, el uso de estos terrenos ha generado otros tipos de conflictos socioambientales.

La educación ambiental juega un rol esencial dentro de este panorama: debe fortalecer la idea de evitar la generación de residuos, poniendo énfasis en la reutilización, el reciclaje y la valorización de esos residuos. Sin embargo, también es relevante hacerse cargo de los residuos sólidos ya dispuestos, y que hoy generan una serie de consecuencias perjudiciales para el medio ambiente y la calidad de vida de las personas. En ese sentido, nos parece fundamental explorar alternativas de recuperación de los suelos contaminados por la basura. El diagnóstico actual es desalentador: «Dos de cada tres municipalidades no tenían acceso a vertederos sanitarios en 2010. Para am- 
pliar el acceso, el Gobierno se propone duplicarlos hasta el año 2020. Sin embargo, un mayor fomento de la prevención de la producción de desechos y del reciclado podría reducir la necesidad de expandir la capacidad existente» (Cepal y OCDE, 2016: 25).

El suelo es un elemento que ha sido dejado de lado por nuestra normativa ambiental, y urge la dictación de una ley que regule la gestión de residuos de una manera integral para evitar la contaminación de los suelos. Pero además de una ley o normativa específica que regule al suelo como un recurso natural no renovable - atendido que su velocidad de formación y regeneración es muy lenta comparada con los procesos que contribuyen a su degradación - es necesario que se determine la existencia de riesgos asociados a los suelos contaminados, así como su magnitud, y se establezcan medidas de recuperación y restauración de suelos con la finalidad de permitir su reutilización, además de políticas y medidas ambientales que garanticen la protección y conservación de los suelos.

El problema de la basura y su impacto en el medio ambiente nos llevan a creer que debemos dar un paso más allá en materia del reciclaje. Hay que tener en cuenta que el 24,25\% de los sitios potencialmente contaminados en nuestro país tienen su origen en la disposición de residuos.

En este contexto, creemos que, si bien es una tecnología aun en desarrollo y poco estudiada, es necesario explorar el landfill mining en nuestro país, con el objetivo de recuperar los suelos y valorizar los residuos ahí dispuestos, evitar la utilización de terrenos adicionales y obtener energía a través del gas metano generado en estos rellenos sanitarios. De hecho, en la actualidad es posible incorporar el landfill mining o la recuperación del suelo a partir del diseño mismo de los vertederos y rellenos sanitarios, para que sean evaluados como una etapa final del proyecto.

\section{Referencias}

Arraiza, María Paz, José Vicente López Álvarez, Belén Vásquez de Quevedo Algora, y Ángela García de Arana (2014). Climate Change and Waste Land Restoration. Technical University of Madrid. Disponible en http://bit.ly/2JwRWaU.

BCN (2019). Los suelos, la agricultura y el cambio climático. Disponible en https:// www.bcn.cl/observatorio/americas/noticias/formulario.2019-01-24.8840620935.

Calderón, Ana Julieth, Paulo Cezar Cassettari Filho, Emília Wanda Rutkowski, Ricardo de Lima Isaac (2019). «Landfill mining as a strategic tool towards global sustainable development». Journal of Cleaner Production, 226.

Cepal y OCDE (2016). Evaluaciones del Desempeño Ambiental: Chile 2016. Disponible en https://bit.ly/2Wbr4Qe.

SINIA, Sistema Nacional de Información Ambiental del Ministerio del Medio Ambiente (2018). Cuarto reporte del estado del medio ambiente. Disponible en https:// bit.ly/3qTRN1U. 
Subdere, Subsecretaría de Desarrollo Regional y Administrativo (2018). «Informe 2. Diagnóstico de la situación por comuna y por región en materia de RSD y asimilables». Disponible en https://bit.ly/2LzwG4Z.

Dubey, A., M. Chakrabarti y D. Pandit (2016). «Landfill mining as a remediation technique for open dumpsites in India». Procedia Environmental Sciences, 35: 319327. Disponible en https://bit.ly/2WfH6sB.

Hogland, William, Marcia Marques y Lars Thorneby (1997). «Landfill Mining: Space Saving, Material Recovery and Energy Use». Waste Management and the Environmental, 41: 339-355. Disponible en https://bit.ly/3gJ9pc8.

Квоок, Joakim, Niclas Svensson y Mats Eklund (2012). «Landfill mining: a critical review of two decades of research». Waste Management, 32: 513-520. Disponible en https://bit.ly/3gONy35.

Ortiz Bernard, Irene, Juana Sanz García, Miriam Dorado Valiño, Susana Villar Fernández (2007). Técnicas de recuperación de suelos contaminados. Madrid: Universidad de Alcalá. Disponible en https://bit.ly/3oUvac5.

UNEP, United Nations Environmental Program (2010). Waste and Climate Change: Global trends and strategy framework. Disponible en https://bit.ly/3mf5EMH.

VAN Der Zee, D.J., M.C. Achterkamp y B. J. Visser. 2004. «Assessing the opportunities of landfill mining». Waste Management, 24: 795-804. Disponible en https:// bit.ly/3a8UZkh.

WRAP, Waste and Resources Action Programme (2013). «Feasibility and viability of landfill mining and reclamation in Scotland». Disponible en https://bit. ly $/ 3$ rK 7 WHB.

Zhou, Chuanbin, Zhe Gong, Junsong Hu, Aixin Cao, Hanwen Liang (2015). A costbenefit analysis of landfill mining and material recycling in China. Waste Management, 35: 191-198.

\section{Agradecimientos}

Agradecemos la colaboración de Andrés Ibarra en la etapa final de escritura de este artículo.

\section{Sobre las autoras}

Constanza Pelayo Díaz es abogada de la Facultad de Derecho de la Universidad de Chile y estudiante del Magíster en Derecho Ambiental de la Universidad del Desarrollo. Su correo electrónico es constanza.pelayo@gmail.com. (D) https://orcid. org/0000-0002-3042-8636. 
Izaskun Linazasoro Espinoza es abogada de la Facultad de Derecho de la Universidad de Chile y candidata a magíster en Derecho con mención en Derecho Público por la misma casa de estudios. Es académica en proceso de nombramiento del Departamento de Derecho Público de la Universidad de Chile y profesora de Derecho Administrativo de la Universidad Católica Silva Henríquez. Su correo electrónico es izaskun.linazasoro@gmail.com. (D) https://orcid.org/0000-0003-4469-9063. 
La Revista de Derecho Ambiental, del Centro de Derecho Ambiental de la Facultad de Derecho de la Universidad de Chile, es un espacio de exposición y análisis en el plano académico del derecho ambiental. Su contenido se presenta a través de doctrina, jurisprudencia y recensiones, y aborda diversas materias relacionadas con la gestión, institucionalidad y herramientas de protección ambiental y desarrollo sustentable. Se presentan artículos de diferentes autores y autoras en los que se analizan y abordan casos y temas jurídico-ambientales de creciente interés y actualidad.

\author{
DIRECTORA \\ Valentina Durán Medina \\ EDITORES \\ Jorge Ossandón Rosales \\ y Antonio Pulgar Martínez \\ SITIO WEB \\ revistaderechoambiental.uchile.cl \\ CORREO ELECTRÓNICO \\ revistada@derecho.uchile.cl \\ LICENCIA DE ESTE ARTÍ́CULO \\ Creative Commons Atribución Compartir Igual 4.o Internacional
}

La edición de textos, el diseño editorial

y la conversión a formatos electrónicos de este artículo

estuvieron a cargo de Tipográfica

(www.tipografica.io) 